\title{
Analysis of high-precision vanadium isotope ratios by medium resolution $\mathrm{MC}$-ICP-MS
}

\author{
Sune G. Nielsen ${ }^{\mathrm{a}, \mathrm{b}, *}$, Jeremy D. Owens ${ }^{\mathrm{a}, \mathrm{b}, 1}$ and Tristan J. Horner ${ }^{\mathrm{a}, \mathrm{c}}$ \\ ${ }^{a}$ NIRVANA laboratories, Woods Hole Oceanographic Institution, Woods Hole, MA, USA \\ ${ }^{\mathrm{b}}$ Department of Geology and Geophysics, Woods Hole Oceanographic Institution, Woods Hole, \\ MA, USA \\ ${ }^{c}$ Department of Marine Chemistry and Geochemistry, Woods Hole Oceanographic Institution, \\ Woods Hole, MA, USA \\ *Corresponding author \\ ${ }^{1}$ Now at: Department of Earth, Ocean and Atmospheric Science, Florida State University, \\ Tallahassee, FL 32306, USA
}




\begin{abstract}
We present and verify a new method to measure vanadium isotope ratios using a Thermo Scientific Neptune multi-collector inductively-coupled plasma mass spectrometer (MCICP-MS) operated in medium mass resolution mode. We collect masses 48 through 53 simultaneously using the L2, L1, Center, H1, H2 and H3 collectors. The Center cup is equipped with a $10^{12} \Omega$ resistor, H1 is equipped with a $10^{10} \Omega$ resistor, while the rest of the collectors have standard $10^{11} \Omega$ resistors. Unlike previous low-resolution methods, the use of medium mass resolution $(\Delta \mathrm{M} / \mathrm{M} \sim 4,000)$ permits separation of $\mathrm{V}$, Ti and $\mathrm{Cr}$ isotopes from all interfering molecular species representing combinations of $\mathrm{C}, \mathrm{N}, \mathrm{O}, \mathrm{S}, \mathrm{Cl}$, and $\mathrm{Ar}$. We show that the external reproducibility follows a power law function with respect to the number of $\mathrm{V}^{+}$ions collected and achieve an external reproducibility of $\pm 0.15 \%$ with total $\mathrm{V}^{+}$ion beam intensities of $\sim 1 \mathrm{nA}$. The separation of interfering molecular species from the $\mathrm{V}$ mass spectrum reduces the $\mathrm{V}$ requirement for precise isotope data to as little as 200-300 ng V per analysis - a reduction of $\sim 90 \%$ compared with previous methods - making several low-V matrices amenable to $\mathrm{V}$ isotope analysis.
\end{abstract}




\section{Introduction}

At chemical equilibrium, the stable isotope fractionation between reactants and products depends on the bond strengths of the molecules on both sides of the reaction ${ }^{1}$. Equilibrium stable isotope fractionation is often greatest for chemical reactions that involve several oxidation states of an element because the bond strengths tend to vary systematically as a function of oxidation state. Therefore, vanadium (V) isotope measurements have great potential as tracers of a wide range of reduction-oxidation (redox) processes because of the four naturally occurring valance states $(+2,+3,+4$, and +5$)$ in different environments on Earth, and potentially other bodies in the solar system ${ }^{2-6}$. Vanadium has two stable isotopes $\left({ }^{50} \mathrm{~V}\right.$ and $\left.{ }^{51} \mathrm{~V}\right)$ with abundances of $0.24 \%$ and 99.76\%, respectively. Theoretical calculations predict that $\mathrm{V}$ isotope fractionation between molecules of $\mathrm{V}$ in different oxidation states should be up to several permil at Earth surface temperatures ${ }^{7}$, but only recently was a method developed that is sufficiently precise and accurate to resolve the predicted natural $\mathrm{V}$ isotope variation ${ }^{8,9}$. Subsequently it has been shown that even rocks formed at temperatures in excess of $1000 \mathrm{~K}$ display resolvable $\mathrm{V}$ isotope differences ${ }^{10}$, which underlines the potential of $\mathrm{V}$ isotopes to track redox processes both in high and low temperature environments.

As is common to other metal isotope systems ${ }^{11}$, measuring $\mathrm{V}$ isotope ratios with high precision and accuracy requires 1) efficient and quantitative separation of the element of interest from sample matrices be obtained and 2) sufficient ion beam signal is produced for the minor $\left({ }^{50} \mathrm{~V}\right)$ isotope in order to achieve acceptable counting statistics for collection of high precision isotope data. Previously described methods for high precision V isotope measurements meet both of these criteria ${ }^{8,9}$ using liquid ion-exchange chromatography to remove isobaric $\mathrm{Ti}$ and $\mathrm{Cr}$ from the sample matrix whilst ensuring quantitative $\mathrm{V}$ recovery ${ }^{8}$. The mass spectrometric protocols of 
the original $\mathrm{V}$ isotope measurement $\operatorname{method}^{8}$, utilizes a $10^{9} \Omega(\mathrm{Ohm})$ resistor on the faraday detector that collects ${ }^{51} \mathrm{~V}$, which allows larger amounts of $\mathrm{V}$ to be introduced into the multicollector inductively-coupled plasma mass spectrometer (MC-ICP-MS). The larger quantities of $\mathrm{V}$ yield sufficient ${ }^{50} \mathrm{~V}$ signals on a standard $10^{11} \Omega$ resistor for collection of high precision data.

However, there are two major drawbacks to the method that uses $10^{9}$ and $10^{11}$ resistors, which can affect the sample diversity that can be investigated and potentially the data quality. First, due to the use of the $10^{9} \Omega$ resistor, it was found that at least $2 \mu \mathrm{g}$ of $\mathrm{V}$ was consumed for each $\mathrm{V}$ isotope analysis ${ }^{8}$. Although many rocks and sediments on Earth contain several hundred $\mu \mathrm{g} / \mathrm{g}$, there are also many environments that are characterized by much lower $\mathrm{V}$ abundances, which makes separation of sufficient amounts of $\mathrm{V}$ for isotope analysis impractical or impossible with current chemical separation protocols. Secondly, the mass spectrum collected for vanadium isotope measurements contains a large array of interfering molecular species (Table 1), which cannot be individually monitored and corrected for in low resolution (LR, low resolution $\Delta \mathrm{M} / \mathrm{M}$ 300). The molecules listed in Table 1 require a mass resolving power of $\Delta M / M=1900-3200$ and, therefore, it has to be assumed that these molecular interferences are either so small that they do not affect $\mathrm{V}$ isotope measurements, or that they are effectively constant between measuring samples and standards. While these assumptions are likely correct for many types of sample, it is exceedingly difficult to identify small (i.e. sub permil) effects from molecular interferences because there is no independent way to monitor them.

Here, we present a new mass spectrometric method using medium resolution mode (MR, $\Delta \mathrm{M} / \mathrm{M} \sim 4,000)$ of a Thermo Scientific Neptune MC-ICP-MS. The setup presented is able to resolve all interfering molecular species in the $\mathrm{V}$ mass spectrum, thus removing the uncertainty of minor isotope effects from combinations of polyatomic $\mathrm{C}, \mathrm{N}, \mathrm{O}, \mathrm{S}, \mathrm{Ar}$ and $\mathrm{Cl}$. The method 
utilizes high-transmission interface cones that significantly lower the amount of $\mathrm{V}$ required per analysis to roughly 200-300ng, or almost an order of magnitude less than previous methods.

\section{Mass spectrometry protocols}

Vanadium isotope compositions are measured using a Thermo Scientific Neptune MC-ICPMS. The front-end is configured with a 'Jet' sampler cone and an ' $\mathrm{X}$ '-type skimmer cone in order to obtain the highest possible $\mathrm{V}$ transmission efficiency. Vanadium is introduced into the mass spectrometer as a dry aerosol by passing the sample through a CETAC Aridus II desolvator, which is fed by an ESI perfluoroalkoxy alkane (PFA) nebulizer that aspirates the sample solution at $\sim 120 \mu \mathrm{l} / \mathrm{min}$. This configuration routinely produces a vanadium ion beam of $\sim 2.5 \mathrm{nA}$ for a solution containing $1 \mu \mathrm{g} / \mathrm{g}$ vanadium when the mass spectrometer is operated in MR, equivalent to a total ion transmission of $\sim 0.7 \%$.

We collect masses 48 through 53 simultaneously using the L2, L1, Center, H1, H2 and H3 collectors (Table 2). The Center cup is equipped with a $10^{12} \Omega$ resistor, H1 is equipped with a $10^{10} \Omega$ resistor, while the rest of the collectors are connected to standard $10^{11} \Omega$ resistors. The setup produces a mass spectrum as shown in Fig. 1a.

Vanadium isotope measurements are otherwise performed according to the protocols outlined by Nielsen et al. ${ }^{8}$. Each sample analysis consists of 30 s background that is measured by defocusing and deflecting the ion beam away from the detectors in the electrostatic analyzer, followed by 40 cycles of $4.194 \mathrm{~s}$ integrations. Correction for interferences on ${ }^{50} \mathrm{~V}$ from ${ }^{50} \mathrm{Ti}$ and ${ }^{50} \mathrm{Cr}$ that cannot be resolved in MR (nor high resolution) mode are performed as outlined in Nielsen et al. ${ }^{8}$. Each sample is bracketed by four (two on each side) measurements of the Alfa Aesar $(\mathrm{AA}-\mathrm{V})$ standard that has been defined as $\delta^{51} \mathrm{~V} \equiv 0$ (where $\delta^{51} \mathrm{~V}=1000 \times\left[\left({ }^{51} \mathrm{~V} /{ }^{50} \mathrm{~V}_{\text {sample }}\right.\right.$ - 
$\left.\left.{ }^{51} \mathrm{~V} /{ }^{50} \mathrm{~V}_{\mathrm{AA}-\mathrm{V})} /{ }^{51} \mathrm{~V} /{ }^{50} \mathrm{~V}_{\mathrm{AA}-\mathrm{V}}\right]\right)$. In addition, each sample measurement is bracketed by two measurements of the BDH vanadium solution that has an isotope composition of $\delta^{51} \mathrm{~V}=-1.19 \pm$ $0.12 \%(2 \mathrm{sd})^{8}$.

We use an autosampler to perform long sequences of $\mathrm{V}$ isotope measurements, which serves to minimize uncertainty that may arise from systematic drift in the instrumental mass bias. Each sample and standard measurement are always spaced by the exact same amount of time, enabling a time-dependent correction of instrumental mass bias.

\section{Tests of mass spectrometric method performance}

In order to investigate the accuracy and precision of the MR protocols we designed a series of tests to determine the sensitivity of measured ${ }^{51} \mathrm{~V} /{ }^{50} \mathrm{~V}$ ratios to several instrumental parameters, which are addressed in the following sections:

1. The effect of measuring at different $m / z$ to assess the proportion of the peak flat that is free from interferences

2. The effect of (mis)-matching $\mathrm{V}$ concentrations between samples and standard solutions

3. The addition of sulfur to samples and artifacts from S-O molecular interferences

4. The optimal V concentration - in terms of precision - at which to perform V isotope measurements to obtain data with precision comparable to that of previously described LR methods

It should be noted that these tests were not intended to investigate effects resulting from inadequate $\mathrm{V}$ separation from sample matrix during liquid ion exchange chromatography, as these are discussed in detail elsewhere? 


\subsection{Width of peak flat and magnet stability}

The width of the peak flat obtained in MR is approximately 0.01 atomic mass units (amu) (Fig. 1a). We position the magnet approximately $1 / 3$ of the way from the left shoulder and keep this mass setting throughout each run sequence. The magnet is positioned towards lower $\mathrm{m} / \mathrm{z}$ to avoid any potential effects from tailing of interference peaks ${ }^{12}$ that all possess higher $m / z$. After each sequence is completed we scan across the mass spectrum to verify that the magnet has not drifted during the run. So far we have not observed the magnet to drift by more than $0.002 \mathrm{amu}$ (20\% of peak width) during sequences of at least 12 hours. We are thus confident that magnet stability is sufficient to avoid effects from magnet drift. As expected, our tests of the peak flat (Fig. 1b and 1c) also showed that tailing from interferences on ${ }^{50} \mathrm{~V}$ that cause a systematic decrease in the measured ${ }^{51} \mathrm{~V} /{ }^{50} \mathrm{~V}$ ratios are the most significant sources of deviation from peak flatness on the high $\mathrm{m} / \mathrm{z}$ side of the peak flat. However, these interference peak tails appear to be similar for standards and samples because no change in the relative isotopic difference between $\mathrm{BDH}$ and $\mathrm{AA}-\mathrm{V}$ was recorded over the entire peak flat (Fig. 1c).

\subsection{Effects from variations in sample and standard V concentrations}

As outlined above, a host of molecular interferences are collected together with the main isotopes of $\mathrm{Ti}, \mathrm{V}$ and $\mathrm{Cr}$ during LR measurements (Table 1). Assuming that these molecular interferences are constant for samples and standards, it was determined that the $\mathrm{V}$ concentrations of samples and bracketing standards had to be matched to better than $15 \%{ }^{8,9}$ in order to normalize away the molecular interferences. However, all molecular interferences across the V mass spectrum are efficiently resolved in $\mathrm{MR}$, suggesting that $\mathrm{V}$ isotope ratios should be 
insensitive to this issue as long as $\mathrm{V}$ is effectively isolated from the sample matrix. To test if this was the case, we performed two tests using (i) matrix-free AA-V standards of differing concentrations and (ii) sample seawater matrix-doped AA-V that was subsequently processed through previously published ion exchange chromatographic procedures ${ }^{8}$. The latter tests were conducted to ascertain if any of the molecular interferences were dependent on residual matrix from geological samples.

The results of the tests with a seawater matrix are shown in Figure 2, illustrating a small but measureable effect for large mismatches in concentration between samples and standards. The effect correlates with the ratio between the $\mathrm{V}$ ion beam intensities of samples and standards $\left(\mathrm{R}^{2}=\right.$ 0.71 ) and shows that $>30 \%$ mismatch results in a systematic offset in $\delta^{51} \mathrm{~V}$ of $\sim 0.15 \%$. This effect is also apparent for standards that have not been doped with sample matrix (Fig. 1c). As such, this effect most likely relates to minor peak tails from interferences on the high $\mathrm{m} / \mathrm{z}$ side of the peak flat that can affect measured ${ }^{51} \mathrm{~V} /{ }^{50} \mathrm{~V}$ ratios, rather than molecular interferences that are present in residual sample matrix. Though these effects are probably smaller than those observed for the LR method ${ }^{8}$, we recommend that samples and standards continue to be matched to within $\pm 15 \%$ in order to eliminate these effects. In addition, it can be seen that the effect is enhanced at lower ion beam intensities (Fig. 1c), which suggests that the interference tail(s) originate from polyatomic molecular species containing $\mathrm{Ar}, \mathrm{O}$, and $\mathrm{N}$, rather than sample matrix. However, the effect may still be enhanced by interferences originating from residual sample matrix (e.g. most likely $\mathrm{S}, \mathrm{C}$, and $\mathrm{Cl}$ ), but based on our present tests (Fig. 2) there is no indication that these contributions are significant. However, it is likely that residual sample matrix left over from V separation depends strongly on sample type processed, such that we encourage future studies to perform $\mathrm{V}$ isotope verification for each type of sample matrix encountered (e.g. seawater, 
organic rich sediments, basalts, granites) that may introduce small, but significant inaccuracies in $\mathrm{V}$ isotope data.

\subsection{Sulfur addition to standard solutions}

Previous $\mathrm{V}$ isotope measurement techniques documented that significant quantities of $\mathrm{S}$ in sample solutions greatly modify measured $\mathrm{V}$ isotope compositions. This effect is due to the apparent increase in Ti and $\mathrm{V}$ ion beams that results from S-O molecular interferences (Table 1). When operating the Neptune MC-ICP-MS at Woods Hole Oceanographic Institution (WHOI) in LR, we observed much larger effects on measured $\mathrm{V}$ isotope ratios from $\mathrm{S}$ addition than was previously observed for the $\mathrm{Nu}$ Plasma (Fig. 3). Given that oxide production rates for the $\mathrm{Nu}$ Plasma and Neptune MC-ICP-MS instruments are similar ${ }^{13}$, the higher S-O interferences observed for the Neptune is likely due to the higher transmission for the Neptune in the low mass end of the periodic table than the $\mathrm{Nu}$ Plasma. Thus, for a solution with a fixed $\mathrm{S}: \mathrm{V}$ ratio, significantly higher $\mathrm{S} / \mathrm{V}$ is measured on the Neptune MC-ICP-MS compared to the Nu Plasma. In turn, this relationship results in higher abundances of S-O interferences on the Neptune (Fig. 3). The published $\mathrm{V}$ separation protocols may result in non-trivial amounts of $\mathrm{S}$ in the isolated $\mathrm{V}$ because no specific part of the chemical separation procedure removes $\mathrm{S}$ quantitatively. We therefore tested the effect of $\mathrm{S}$ addition to BDH solutions with known $\delta^{51} \mathrm{~V}$ of $-1.19 \pm 0.12 \%$ o (2sd) by adding enough sulfur to obtain $\mathrm{S} / \mathrm{V} \sim 2$. Vanadium isotope measurements in LR with half the $\mathrm{S} / \mathrm{V}$ ratio revealed offsets from the true value of up to several permil (Fig. 3). However, the results from this test in $\mathrm{MR}$ show that - even at low $\mathrm{V}$ ion beam intensities - there is no detectable effect on measured V isotope composition (Fig. 4b). 


\subsection{Optimal V concentration for samples and standards}

Several of the mass spectrometric tests that use very low $\mathrm{V}$ ion beam intensities (Fig. 1 and 2) display precision that is slightly inferior $( \pm 0.2 \%$ vs. $\pm 0.12 \%$ ) to that previously achieved for $5 \mu \mathrm{g} / \mathrm{g}$ solutions in LR mode with a Nu Plasma MC-ICP-MS ${ }^{8}$. We have tested the uncertainty on the $\mathrm{BDH}$ standard measurements as a function of ${ }^{51} \mathrm{~V}$ ion beam intensity and find that individual sequences produce uncertainties on repeat $\mathrm{BDH}$ measurements better than or equal to $\pm 0.12 \%$ o only when ${ }^{51} \mathrm{~V}$ ion beam intensity is greater than $\sim 1.5 \mathrm{nA}$ (Fig. 4a). In addition, the uncertainty generally follows a power law whereby higher ion beam intensities are associated with lower uncertainties. However, the slope of the power law we derive from measurements of BDH is approximately -0.9 (Fig. 4a), which is significantly steeper than the slope of -0.5 expected if all uncertainties were explained by counting statistics ${ }^{14}$. Similar to effects observed for iron isotope measurements using standard-sample bracketing ${ }^{14}$, we conclude that uncertainties on V isotope measurements have a significant component from other sources of uncertainty, such as instability of the plasma, that are most important at low ion beam intensities. These uncertainties only apply to pure vanadium standard solutions and are likely slightly larger for samples, as has been previously reported ${ }^{9,10}$. However, with our routine running conditions where we obtain $\mathrm{V}$ ion currents of $\sim 1.5 \mathrm{nA}$ total $\mathrm{V}$ for solutions containing $0.6 \mu \mathrm{g} / \mathrm{g}$ vanadium, we are able to achieve $\pm 0.12 \%$ (Fig. 4), which consumes only $\sim 260$ ng of $\mathrm{V}$ per analysis (30 sec background, $20 \mathrm{sec}$ idle time, $168 \mathrm{sec}$ analysis time). This amount of $\mathrm{V}$ is almost an order of magnitude less than required by the previous LR method using a Nu Plasma MC-ICP-MS. Two of the major reasons for this reduction in sample-derived $\mathrm{V}$ is that (i) previous methods utilized low-transmission sampler and skimmer cones to minimize the impact of molecular interferences across the vanadium mass spectrum ${ }^{8}$ and (ii) previous methods used tuning parameters that were set to 
minimize molecular interferences, by employing low $\mathrm{Ar}$ gas flows across the desolvating membrane, and utilizing high RF power to break down molecular interferences. Both of these tuning parameters result in variably lower $\mathrm{V}$ transmission than if tuning for optimum $\mathrm{V}$ transmission efficiency as we do here.

\section{Conclusions}

We have developed and verified a new medium resolution mass spectrometric protocol to measure high precision V isotope ratios using a Thermo Scientific Neptune MC-ICP-MS. The method achieves a comparable precision comparable to previous LR methods of $\pm 0.12 \%$, while consuming as little as $260 \mathrm{ng}$ of $\mathrm{V}$-almost an order of magnitude less V per analysis. Given the practical limits of ion-exchange chromatographic separation procedures that cannot more than $100 \mathrm{mg}$ of matrix per resin column, this $\sim 90 \%$ reduction in $\mathrm{V}$ requirements by using MR will significantly aid future studies in obtaining precise $\mathrm{V}$ isotope data for samples with low $\mathrm{V}$ concentrations that were otherwise difficult or impractical to measure. For example, this new method makes many types of previously inaccessible samples with low V contents amenable to $\mathrm{V}$ isotope analysis, such as seawater, depleted peridotites, iron meteorites, carbonates, and biomass, which are all of significant interest across the fields of Earth and Environmental Sciences.

\section{Acknowledgements}

We thank Dr. Jerzy Blusztajn for his tireless help in the WHOI plasma mass spectrometer facility. This study was funded by a WHOI IR\&D grant to SGN and NSF OCE grant 1434785 to JDO and SGN and Agouron Geobiology Fellowships to JDO and TJH. 


\section{References}

1. H. C. Urey, J. Chem. Soc., 1947, 562-581.

2. J. L. Morford and S. Emerson, Geochim. Cosmochim. Acta., 1999, 63, 1735-1750.

3. B. J. Wood, J. Wade and M. R. Kilburn, Geochim. Cosmochim. Acta., 2008, 72, $1415-$ 1426.

4. K. Righter, S. R. Sutton, M. Newville, L. Lei, C. S. Schwandt, H. Uchida, B. Lavina and R. T. Downs, American Mineralogist, 2006, 91, 1643-1656.

5. B. Wehrli and W. Stumm, Geochim. Cosmochim. Acta., 1989, 53, 69-77.

6. M. D. Lewan and J. B. Maynard, Geochim. Cosmochim. Acta., 1982, 46, 2547-2560.

7. F. Wu, T. Qin, X. F. Li, Y. Liu, J. H. Huang, Z. Q. Wu and F. Huang, Earth Planet. Sci. Lett., 2015, 426, 216-224.

8. S. G. Nielsen, J. Prytulak and A. N. Halliday, Geostand. Geoanal. Res., 2011, 35, 293306.

9. J. Prytulak, S. G. Nielsen and A. N. Halliday, Geostand. Geoanal. Res., 2011, 35, 307318.

10. J. Prytulak, S. G. Nielsen, D. A. Ionov, A. N. Halliday, J. Harvey, K. A. Kelley, Y. Niu, D. W. Peate, K. Shimizu and K. W. W. Sims, Earth Planet. Sci. Lett., 2013, 365, $177-$ 189.

11. C. M. Johnson, B. L. Beard and F. Albarede, Rev Mineral Geochem, 2004, 55, 1-24.

12. S. Weyer and J. Schwieters, Int. J. Mass Spectrom., 2003, 226, 355-368.

13. J. L. Payne, N. J. Pearson, K. J. Grant and G. P. Halverson, J. Anal. At. Spectrom., 2013, 28, 1068-1079.

14. S. G. John and J. F. Adkins, Mar. Chem., 2010, 119, 65-76. 


\section{Figure captions}

Figure 1: a) Mass scan of the AA-V standard in medium resolution mode across the interference free portion of the mass spectrum measured. Note that $\mathrm{m} / \mathrm{z}=49$ and $\mathrm{m} / \mathrm{z}=53$ are not shown because they are so small for AA-V that the ion beams are primarily noise. b) Vanadium isotope measurements of the AA-V standard across the portion of the mass spectrum in a) (from 49.92 to $49.93 \mathrm{amu}$ ) that visually appears free of interferences. Measurements were performed using four different ion beam intensities. Peak tails of molecular interferences on ${ }^{50} \mathrm{~V}$ cause the lower ${ }^{51} \mathrm{~V} /{ }^{50} \mathrm{~V}$ recorded towards the right side of the mass spectrum. c) AA-V standard solutions of different vanadium ion beam intensities measured against an AA-V solution that yielded $\sim 0.67 \mathrm{nA}^{51} \mathrm{~V}$ across the portion of the mass spectrum in a) (from 49.92 to $49.93 \mathrm{amu}$ ) that visually appears free of interferences. Also shown are measurements of the BDH vanadium solution against AA-V across the same portion of the mass spectrum. The ion beam intensity of the $\mathrm{BDH}$ and bracketing AA-V solutions were $\sim 0.67 \mathrm{nA}$ and were matched to within $4 \%$.

Figure 2: Vanadium isotope compositions measured for four separate seawater matrix-doped splits of AA-V plotted against the ratio of the ion beam intensity for the processed AA-V and the bracketing unprocessed AA-V standards. Shading denotes the areas where samples and standards are matched to better than $\pm 30 \%$ and $\mathrm{V}$ isotope compositions are within $\pm 0.15 \%$ of the true value (三0 \%). The seawater matrix was removed from each AA-V split using ion exchange chromatography. Different colored symbols denote different splits of AA-V that were put through the column chemistry separation procedure. When sample ion beam intensities are more than $30 \%$ different to the bracketing standards, $\mathrm{V}$ isotope compositions recorded deviate from the 
true value by more than $\pm 0.15 \%$. This effect is similar to that observed for pure standards (Fig. 1c) suggesting that the $\mathrm{V}$ isotope artifact does not originate from residual sample matrix.

Figure 3: Vanadium isotope compositions measured for $\mathrm{BDH}$ vanadium solutions doped with variable amounts of sulfur plotted against the recorded ratio of $m / z-49 / m / z-51$. Measurements performed in both low and medium resolution on the Neptune MC-ICP-MS are displayed. Also shown are similar tests performed on the $\mathrm{Nu}$ Plasma $^{8}$ albeit with significantly greater amounts of sulfur added. Grey bars denote the value with 2sd error envelope of undoped $\mathrm{BDH}$ vanadium solutions.

Figure 4: a) Two standard deviation uncertainties on repeat measurements of BDH vanadium solutions in medium resolution as a function of the vanadium ion beam intensity. Each data point represents between 26 and 43 individual analyses of BDH. b) Vanadium isotope composition of six repeat analyses of BDH vanadium solution doped with sulfur (red circle; S/V 2). Error bars are 2sd. Also shown are the undoped BDH vanadium measurements plotted in a). The grey envelope denotes the fitted uncertainty from a) associated with measurements of undoped $\mathrm{BDH}$ vanadium solutions in medium resolution. 
Table 1: Molecular species resolved in medium resolution

\begin{tabular}{|c|c|c|c|}
\hline $\begin{array}{l}\text { Molecular } \\
\text { species* }\end{array}$ & $\begin{array}{c}\text { Species } \\
\text { abundance }\end{array}$ & $\begin{array}{c}\text { Reference } \\
\text { isotope }\end{array}$ & $\Delta \mathrm{M} / \mathrm{M}$ \\
\hline${ }^{32} \mathrm{~S}-{ }^{16} \mathrm{O} 6$ & 0.94793 & ${ }^{48} \mathrm{Ti}$ & 2518 \\
\hline${ }^{36} \mathrm{Ar}-{ }^{12} \mathrm{C}$ & 0.00333 & ${ }^{48} \mathrm{Ti}$ & 2446 \\
\hline${ }^{36} \mathrm{~S}-{ }^{12} \mathrm{C}$ & 0.00019 & ${ }^{48} \mathrm{Ti}$ & 2506 \\
\hline${ }^{34} \mathrm{~S}-{ }^{14} \mathrm{~N}$ & 0.04194 & ${ }^{48} \mathrm{Ti}$ & 2085 \\
\hline${ }^{33} \mathrm{~S}-{ }^{15} \mathrm{~N}$ & 0.00002 & ${ }^{48} \mathrm{Ti}$ & 2030 \\
\hline${ }^{35} \mathrm{Cl}-{ }^{13} \mathrm{C}$ & 0.00833 & ${ }^{48} \mathrm{Ti}$ & 1976 \\
\hline${ }^{37} \mathrm{Cl}^{12} \mathrm{C}$ & 0.23954 & ${ }^{49} \mathrm{Ti}$ & 2715 \\
\hline${ }^{33} \mathrm{~S}^{16} \mathrm{O}$ & 0.00748 & ${ }^{49} \mathrm{Ti}$ & 2646 \\
\hline${ }^{34} \mathrm{~S}^{15} \mathrm{~N}$ & 0.00015 & ${ }^{49} \mathrm{Ti}$ & 2435 \\
\hline${ }^{36} \mathrm{Ar}^{13} \mathrm{C}$ & 0.00004 & ${ }^{49} \mathrm{Ti}$ & 2125 \\
\hline${ }^{32} \mathrm{~S}^{17} \mathrm{O}$ & 0.00036 & ${ }^{49} \mathrm{Ti}$ & 2098 \\
\hline${ }^{35} \mathrm{Cl}^{14} \mathrm{~N}$ & 0.75503 & ${ }^{49} \mathrm{Ti}$ & 2035 \\
\hline${ }^{38} \mathrm{Ar}^{12} \mathrm{C}$ & 0.00062 & ${ }^{50} \mathrm{~V}$ & 3208 \\
\hline${ }^{34} \mathrm{~S}^{16} \mathrm{O}$ & 0.04200 & ${ }^{50} \mathrm{~V}$ & 3198 \\
\hline${ }^{35} \mathrm{Cl}^{15} \mathrm{~N}$ & 0.00278 & ${ }^{50} \mathrm{~V}$ & 2291 \\
\hline${ }^{37} \mathrm{Cl}^{13} \mathrm{C}$ & 0.00266 & ${ }^{50} \mathrm{~V}$ & 2261 \\
\hline${ }^{36} \mathrm{~S}^{14} \mathrm{~N}$ & 0.00020 & ${ }^{50} \mathrm{~V}$ & 2172 \\
\hline${ }^{36} \mathrm{Ar}^{14} \mathrm{~N}$ & 0.00336 & ${ }^{50} \mathrm{~V}$ & 2129 \\
\hline${ }^{32} \mathrm{~S}^{18} \mathrm{O}$ & 0.00190 & ${ }^{50} \mathrm{~V}$ & 2075 \\
\hline${ }^{35} \mathrm{Cl}^{16} \mathrm{O}$ & 0.75600 & ${ }^{51} \mathrm{~V}$ & 2572 \\
\hline${ }^{34} \mathrm{~S}^{17} \mathrm{O}$ & 0.00002 & ${ }^{51} \mathrm{~V}$ & 2212 \\
\hline${ }^{36} \mathrm{Ar}^{15} \mathrm{~N}$ & 0.00001 & ${ }^{51} \mathrm{~V}$ & 2150 \\
\hline${ }^{37} \mathrm{Cl}^{14} \mathrm{~N}$ & 0.24131 & ${ }^{51} \mathrm{~V}$ & 2037 \\
\hline${ }^{33} \mathrm{~S}^{18} \mathrm{O}$ & 0.00002 & ${ }^{51} \mathrm{~V}$ & 1911 \\
\hline${ }^{36} \mathrm{~S}^{16} \mathrm{O}$ & 0.00020 & ${ }^{52} \mathrm{Cr}$ & 2418 \\
\hline${ }^{40} \mathrm{Ar}^{12} \mathrm{C}$ & 0.98504 & ${ }^{52} \mathrm{Cr}$ & 2375 \\
\hline${ }^{36} \mathrm{Ar}^{16} \mathrm{O}$ & 0.00336 & ${ }^{52} \mathrm{Cr}$ & 2366 \\
\hline${ }^{38} \mathrm{Ar}^{14} \mathrm{~N}$ & 0.00063 & ${ }^{52} \mathrm{Cr}$ & 2053 \\
\hline${ }^{37} \mathrm{Cl}^{15} \mathrm{~N}$ & 0.00089 & ${ }^{52} \mathrm{Cr}$ & 2037 \\
\hline${ }^{34} \mathrm{~S}^{18} \mathrm{O}$ & 0.00008 & ${ }^{52} \mathrm{Cr}$ & 1959 \\
\hline${ }^{35} \mathrm{Cl}^{17} \mathrm{O}$ & 0.00029 & ${ }^{52} \mathrm{Cr}$ & 1891 \\
\hline${ }^{37} \mathrm{Cl}^{16} \mathrm{O}$ & 0.24162 & ${ }^{53} \mathrm{Cr}$ & 2626 \\
\hline${ }^{40} \mathrm{Ar}^{13} \mathrm{C}$ & 0.01096 & ${ }^{53} \mathrm{Cr}$ & 2111 \\
\hline${ }^{35} \mathrm{Cl}^{18} \mathrm{O}$ & 0.00152 & ${ }^{53} \mathrm{Cr}$ & 1935 \\
\hline
\end{tabular}

*Only molecular species with an abundance of more than $0.001 \%$ of the total species considered 
Table 2: Isotope abundances in $\%$ of measured $\mathrm{Ti}, \mathrm{V}$ and $\mathrm{Cr}$ isotopes

\begin{tabular}{ccccccc}
\hline Mass (amu) & $\mathbf{4 8}$ & $\mathbf{4 9}$ & $\mathbf{5 0}$ & $\mathbf{5 1}$ & $\mathbf{5 2}$ & $\mathbf{5 3}$ \\
\hline $\mathrm{Ti}$ & 73.8 & 5.51 & 5.34 & & & \\
$\mathrm{~V}$ & & & 0.24 & 99.76 & & \\
$\mathrm{Cr}$ & & & 4.31 & & 83.76 & 9.55 \\
\hline Resistor $(\Omega)$ & $10^{11}$ & $10^{11}$ & $10^{12}$ & $10^{10}$ & $10^{11}$ & $10^{11}$ \\
\hline
\end{tabular}



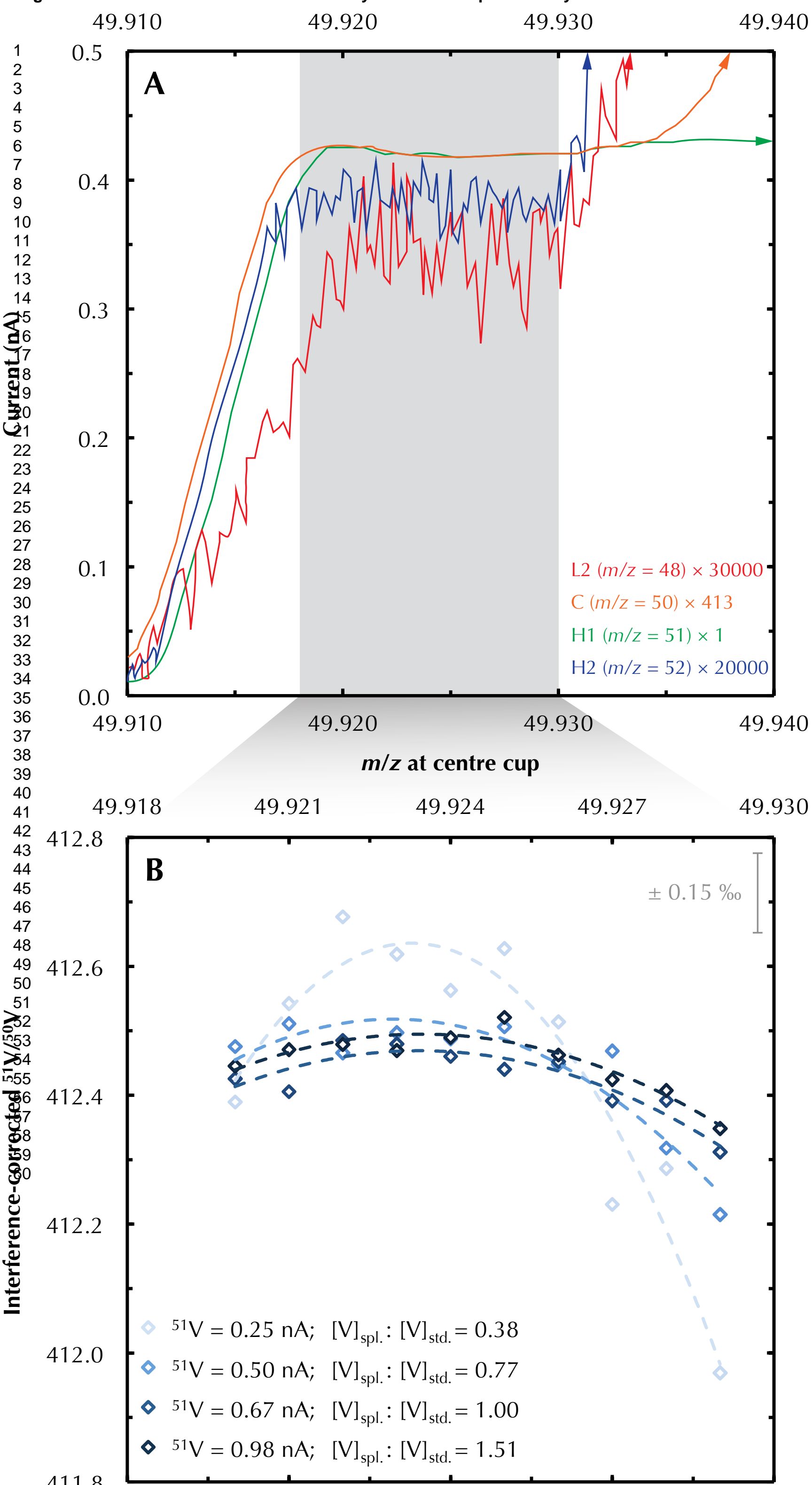

411.8

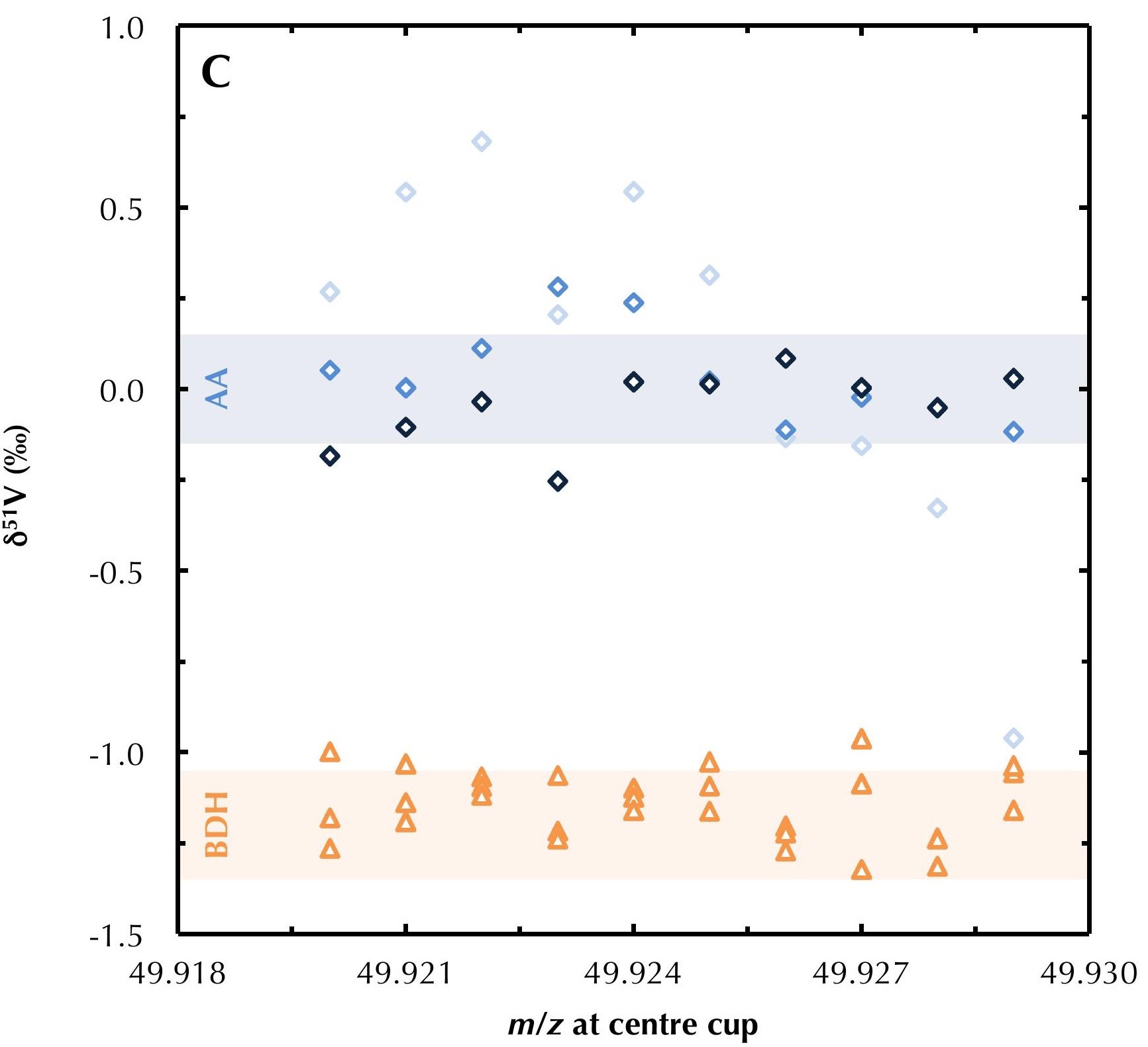




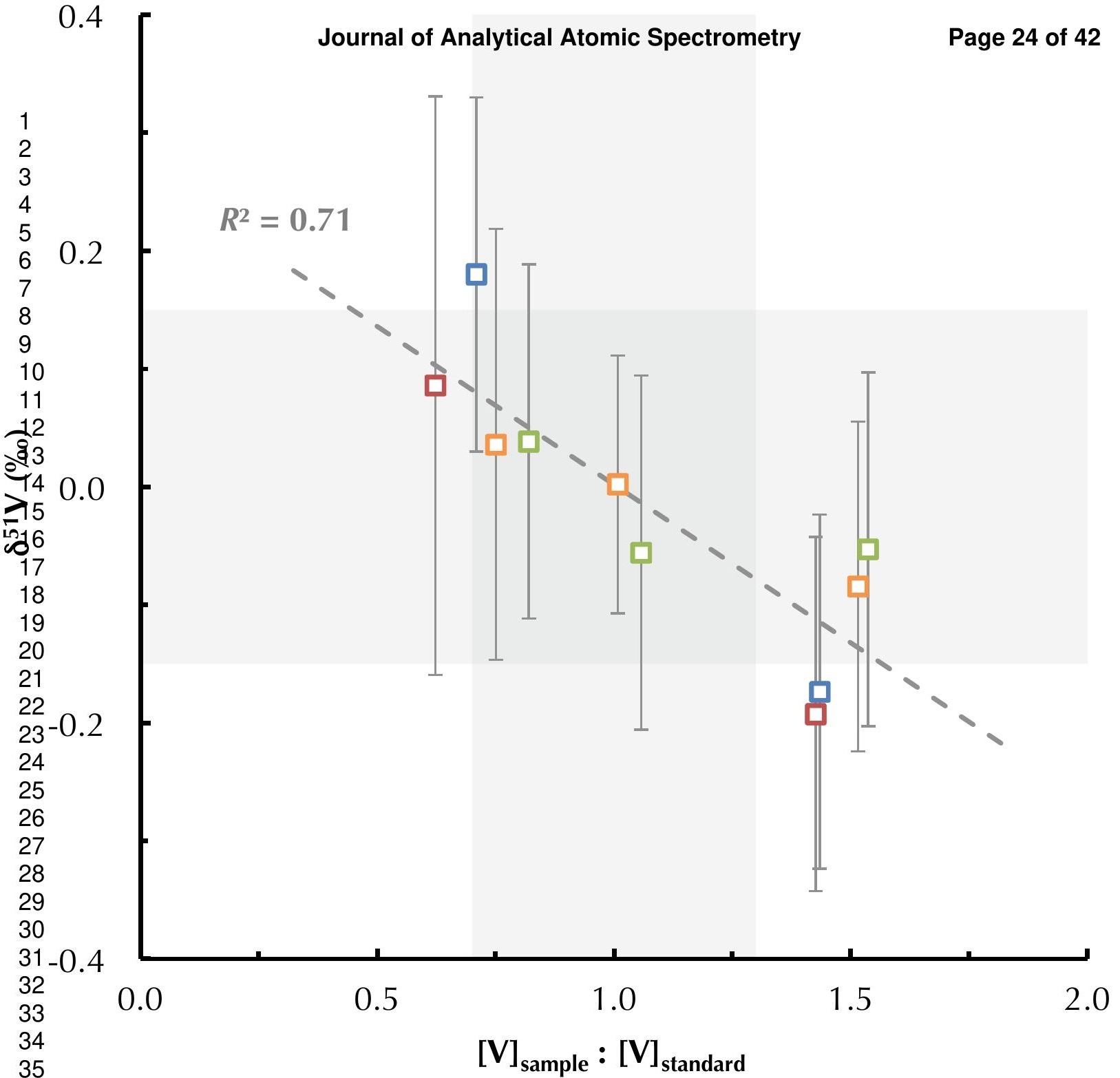




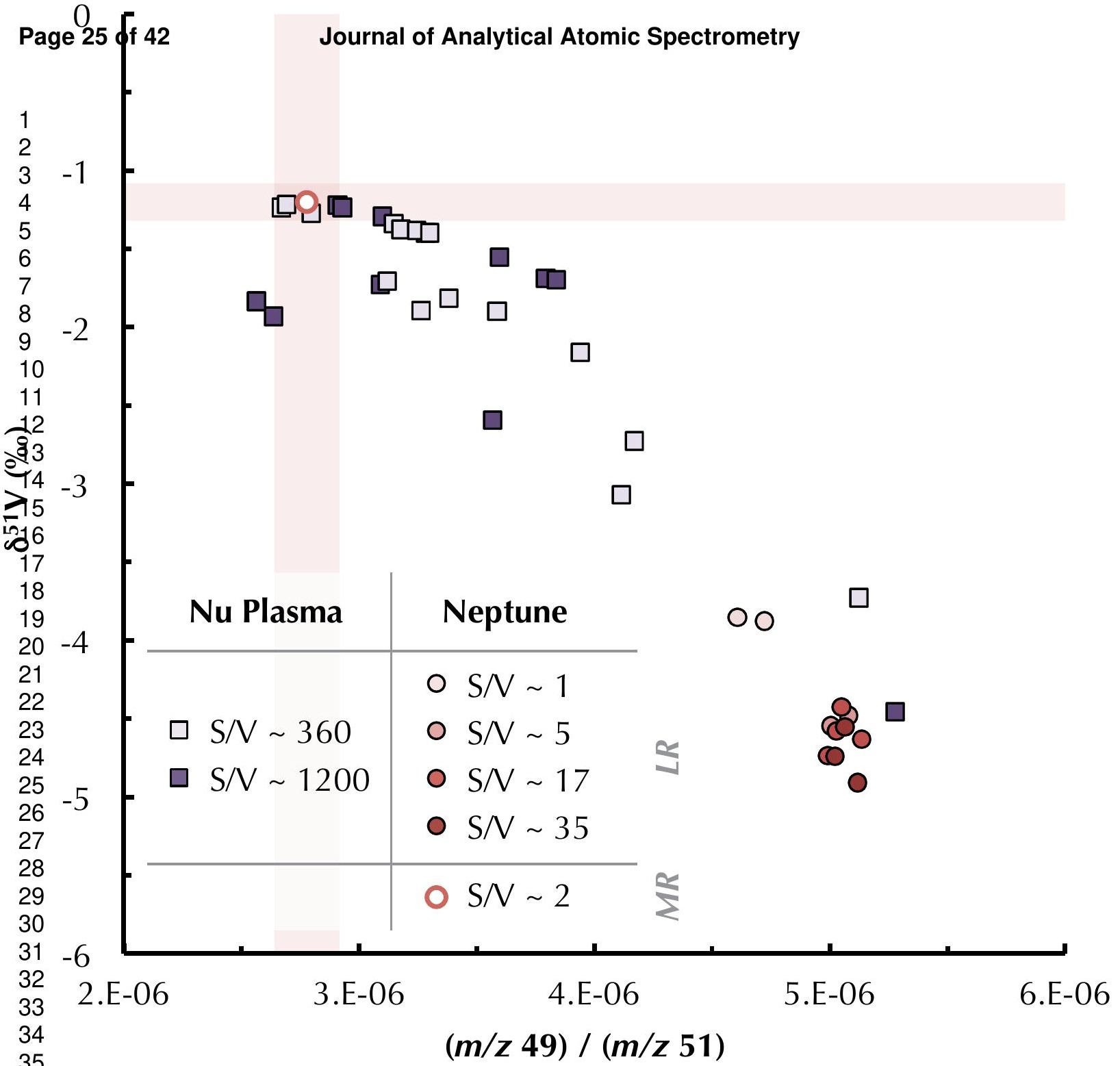



0.4
0.8
1.2

1.6

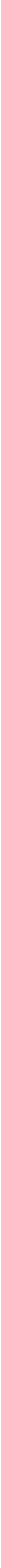

Current on $m / z=51(n A)$ 